\title{
Access optimization in an urban area: a case study
}

\author{
N. Filip, M. Moldovan \& C. Golgot \\ Technical University of Cluj-Napoca, Romania
}

\begin{abstract}
This paper presents a case study of access optimisation for an airport situated in an urban area. Due to the fact that a national road is located near the airport, the access to and from the airport represents a problem. A research programme was initiated to optimise the access to the airport without disrupting the traffic flow in the national road. Two pieces of SDR equipment were used to evaluate the vehicular traffic characteristics including mean speed. Five days of continuous measurements were undertaken to obtain traffic density, flow rate and speed for different types of vehicles.

The proposed solutions were studied to optimise the access, i.e. the effect of a traffic roundabout placed near the airport and the performance of traffic signals with adaptive controls.

The two proposals were analysed using the Syncro 7 program. The study concludes that the best solution is the use of traffic signals with adaptive control, which minimises the delay time for the vehicles running on the national road. In this manner, access to the airport is ensured with a minimum delay (of around 20 seconds) for the case of maximum vehicular flow. The traffic roundabout on the other hand provides better access to the airport, but the time delay for vehicles on the national road increases.

The study was developed upon request from the city council of Cluj-Napoca, Romania.
\end{abstract}

Keywords: vehicle count, roundabout, adaptive traffic lights, vehicle flow, speed, traffic safety. 


\section{Introduction}

Optimisation of vehicular flow in the case of junctions with major roads is still a challenge, particularly in urban areas. One solution is the use of roundabouts in road intersections, which tend to reduce the speed of the vehicles.

The effects of roundabouts have been studied using structural analyses and models. Akçelik in 1991 [1] proposed a model to calculate the effect of one-lane roundabouts, based on macroscopic traffic parameters. Most roundabout studies refer to the work of Kimber and Hollis in 1979 [2] which examines the effect of the number of input lines that meet at the roundabout.

Estimates of traffic flow capacity at roundabouts were carried out at different locations by a series of authors [3-6]. The issue of the general assessment of the roundabout's efficiency and its correlation with traffic safety was studied by Briton in 2005 [7].

An open question is the unmarked intersections of left turns (in countries driving on the right) which may contribute to a traffic hazard giving rise to accidents [8].

Another way to optimise the intersections is the use of time-variable traffic lights that depend on the traffic demand. Simulation studies have been carried out for this type of traffic lights [9-11].

The use of traffic lights to optimise the vehicular flow allows for the orderly displacement of vehicles in platoons interphasing with safe pedestrian crossings. The vehicle movements can be analysed according to the ratio between the main road flow and the total flow, resulting in the same phase number as the number of conflicting phase travel directions.

In the case of traffic lights with fixed phases, an estimate of this ratio is applied, which ought to be evaluated apart against the traffic variation during the day.

The case of increases in flow, leading to saturation, is becoming increasingly common. In this case it is necessary to develop further traffic light phases plans to meet the specific conditions. The new generation of traffic lights - with adaptive functions - allows the green phase time to vary depending on the waiting queue.

In extreme cases, the adaptive system can become on demand traffic lights. The phases are governed by the presence of vehicles. This results in variable green time in different directions, leading to better traffic flow at the junction. The system requires appropriate and robust detection sensors and techniques.

Adaptive traffic lights have the following properties:

- $\quad$ Facilitating the real-time management of heavy traffic.

- Reducing the risk of accidents at junctions with complex geometry or in areas with poor visibility.

This paper presents a case study of the optimisation of a junction without traffic lights between the E60 Romanian national road and an airport access road in the city of Cluj-Napoca. In order to arrive at the best solution the two optimisation techniques already mentioned were applied. 


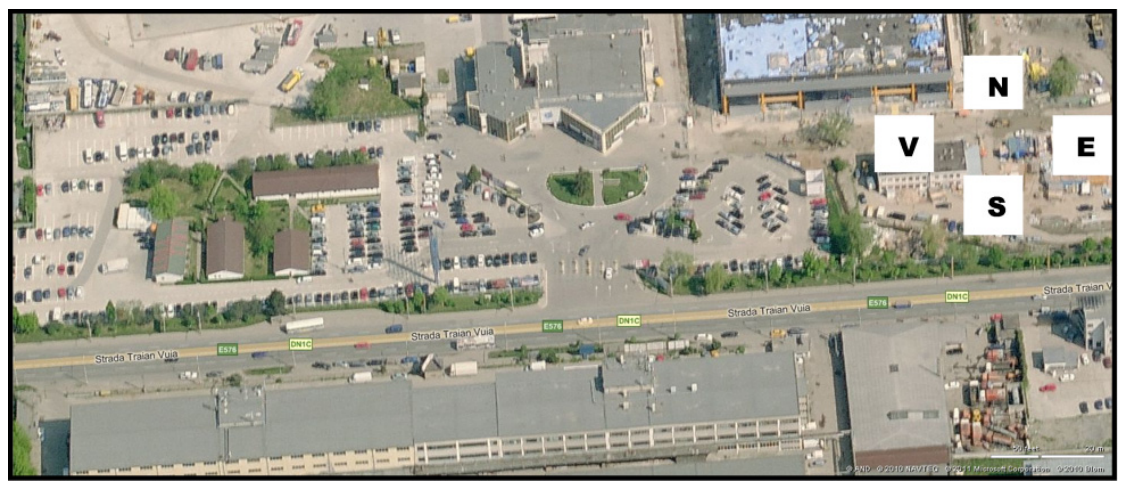

Figure 1: The airport location in suburban area of Cluj-Napoca.

\section{Case study: description of the site}

The airport is located in a suburban area, about $9 \mathrm{~km}$ from downtown ClujNapoca and next to the E60 national road, as shown in Figure 1.

The national road is used by a wide variety of vehicles ranging from bicycles to public transport buses and trucks. It is the eastern access road into ClujNapoca. The road configuration in the airport area has two traffic lanes in each direction, ranging from $3.25 \mathrm{~m}$ to $3 \mathrm{~m}$ wide. A special feature of the vehicle movement in this section of the road is that $70 \%$ of them use the outside traffic lane, with the inside one dedicated to slow vehicles.

The airport access road has three lanes in each direction and meets the E60 at a right angle. Left turns to enter the airport are made with the principle of priority to traffic flow from the right. Presently a STOP traffic sign warns vehicles leaving the airport and entering the main road.

There is no public transportation service for the airport and therefore all passengers arrive and depart the site using private cars or taxis. The current number of daily flights departing and arriving is between 32 and 41, at all hours of day and night (see Figure 2).

The increasing demand for air transport and the large numbers of road accidents recorded around the airport motivated the local authorities to initiate the present study [13].

The following steps were carried out to evaluate the traffic conditions and help develop feasible proposals for airport access optimisation.

- A campaign of continuous traffic monitoring was carried out over 5 working days (20-24 June 2011) on the airport access road, leaving aside the weekend where the traffic is not representative;

- The evaluation of the data collected by the campaign;

- Proposing alternative solutions to optimise airport access;

- Simulation of these proposals using the Syncro 7 traffic simulation system;

- Simulating alternative analyses; 


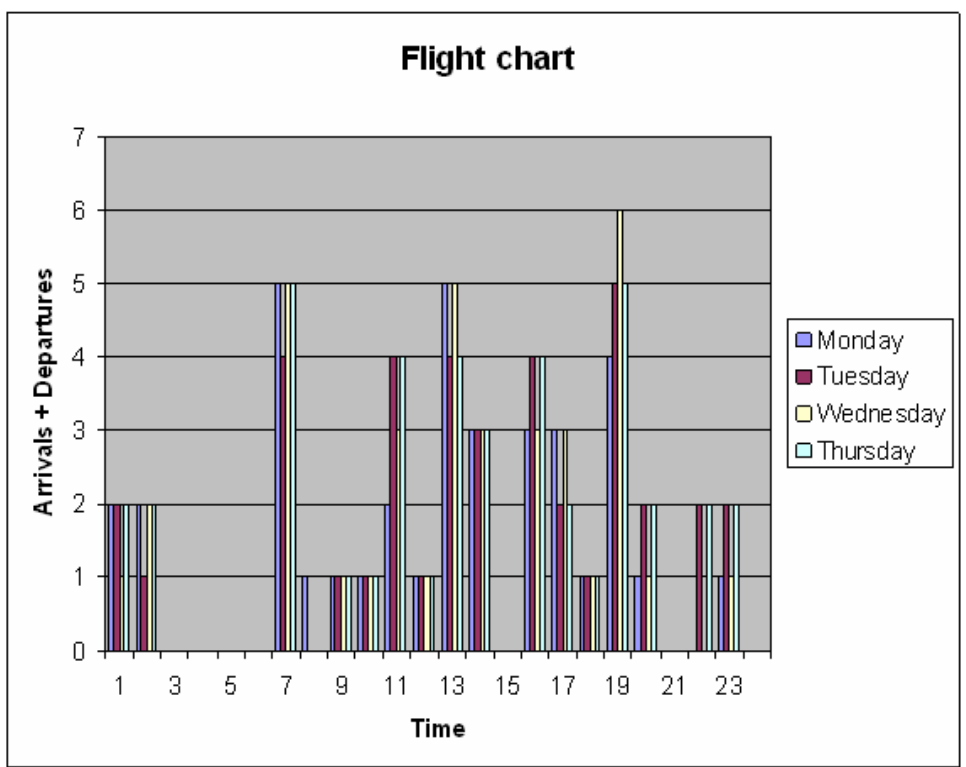

Figure 2: $\quad$ Flight chart for Cluj-Napoca airport.

The optimisation proposals took into consideration the following demands.

- Increase of road safety at the junction;

- Optimise the vehicle flow on the main road;

- Design the site.

\section{Collection and analysis of the traffic data}

\subsection{Traffic data collection}

The five working days' continuous traffic monitoring was carried out using SDR equipment and set to record the presence of a vehicle, vehicle speed and type of road user [4].

The SDR devices were placed in the field as shown in Figure 3, at 120m from the median axis of the airport access road.

The recorded data was uploaded into the Collect Traffic Data Systems program [14] and processed to provide information on time variation of vehicle flow and the velocity field as shown in Figure 4.

The recorded speeds in the main artery, i.e. the national road, were sometimes above the legal limit.

\subsection{Evaluation of traffic data}

Measurements performed on the main road allowed the determination of the number of vehicles that accessed the road from and to the airport. 


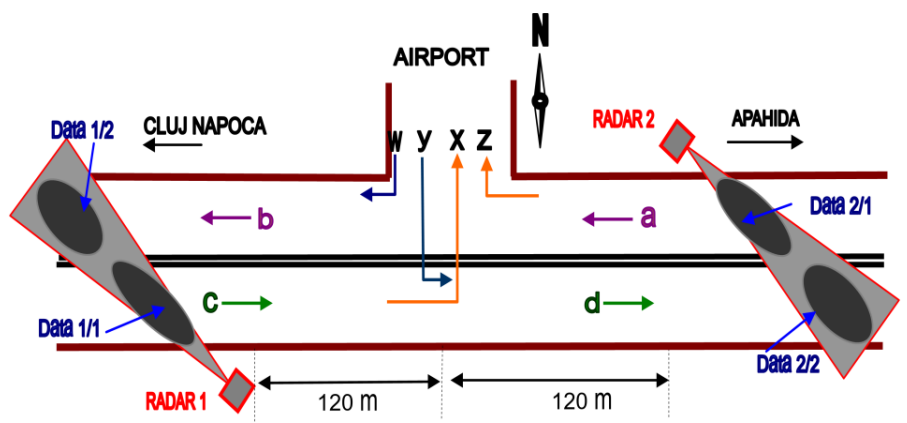

Figure 3: The radar classifier positions for counting.

For access calculations, the following relations were proposed.

$$
\begin{aligned}
& c-d=x+y \\
& b-a=w+z
\end{aligned}
$$

where

c - W-E traffic flow before airport entry on the $\mathrm{W}-\mathrm{E}$ direction,

$\mathrm{d}-\mathrm{E}-\mathrm{W}$ traffic flow behind airport entry on the $\mathrm{W}-\mathrm{E}$ direction,

a - traffic flow before airport entry on the $\mathrm{E}-\mathrm{W}$ direction,

d- traffic flow behind airport entry on the $\mathrm{E}-\mathrm{W}$ direction,

$\mathrm{x}-\mathrm{W}-\mathrm{N}$ entry traffic flow to the airport (turning left),

$y-N-E$ exist traffic flow from the airport (turning left),

$\mathrm{Z}-\mathrm{E}-\mathrm{N}$ entry traffic flow to the airport (turning right),

w- N-W exit traffic flow from the airport (turning right).

The balance equation is obtained by adding the above two equations (1). This represents the total level of airport road access (i.e. in and out):

$$
c-d+b-a=x+y+w+z
$$

The result was a total number of accesses of 4834 vehicles/day, with an average rate of 201 vehicles/hour. The level of access varies between 23 to 468 vehicles per hour.

The typical values of the traffic carried out by the main road are presented in Table 1.

As can be seen in Figure 4, the variation of the vehicle flow is similar for all the five days with the maximum at regular time intervals. Comparing these values with the traffic on the main road, it was found that there are three periods from 7.00 to 18.00 hours when the traffic flow is highly disrupted by that accessing or exiting the airport. 
Table 1: $\quad$ The traffic characteristic for observation days.

\begin{tabular}{|c|c|c|c|c|c|c|c|c|}
\hline \multirow{2}{*}{ Direction } & \multirow{2}{*}{ Day } & \multicolumn{3}{|c|}{ Flow vehicle } & \multicolumn{2}{c|}{ Mean speed } & \multicolumn{2}{c|}{ Traffic participants } \\
\cline { 3 - 10 } & & $24 \mathrm{~h}$ & $\max$ & $\min$ & $\max$ & $\min$ & $\%$ Car & \%Heavy \\
\hline \multirow{4}{*}{$\mathrm{c}(\mathrm{W}-\mathrm{E})$} & $\mathrm{Tu}$ & 15468 & 1093 & 128 & 60 & 53 & 72.61 & 6.03 \\
\cline { 2 - 9 } & $\mathrm{We}$ & 15587 & 1057 & 138 & 62 & 52 & 72.14 & 5.58 \\
\cline { 2 - 9 } & $\mathrm{Thu}$ & 15980 & 1090 & 158 & 60 & 43 & 72.88 & 5.73 \\
\hline \multirow{5}{*}{$\mathrm{d}(\mathrm{W}-\mathrm{E})$} & $\mathrm{Tu}$ & 13868 & 988 & 181 & 62 & 54 & 71.19 & 7.48 \\
\cline { 2 - 9 } & $\mathrm{We}$ & 13807 & 943 & 80 & 61 & 54 & 70.36 & 6.93 \\
\cline { 2 - 9 } & $\mathrm{Thu}$ & 14163 & 915 & 110 & 61 & 53 & 69.04 & 7.03 \\
\hline \multirow{3}{*}{$\mathrm{a}(\mathrm{E}-\mathrm{W})$} & $\mathrm{Tu}$ & 13486 & 1048 & 68 & 55 & 47 & 70.91 & 5.40 \\
\cline { 2 - 9 } & $\mathrm{We}$ & 13060 & 1030 & 78 & 56 & 48 & 72.19 & 5.55 \\
\cline { 2 - 9 } & $\mathrm{Thu}$ & 13501 & 1072 & 64 & 56 & 46 & 72.43 & 5.39 \\
\hline \multirow{3}{*}{$\mathrm{b}(\mathrm{E}-\mathrm{W})$} & $\mathrm{Tu}$ & 14748 & 1025 & 64 & 67 & 60 & 66.21 & 7.42 \\
\cline { 2 - 9 } & $\mathrm{We}$ & 14681 & 992 & 80 & 69 & 57 & 64.66 & 6.88 \\
\cline { 2 - 8 } & $\mathrm{Thu}$ & 15148 & 1033 & 99 & 68 & 58 & 65.03 & 6.68 \\
\hline
\end{tabular}
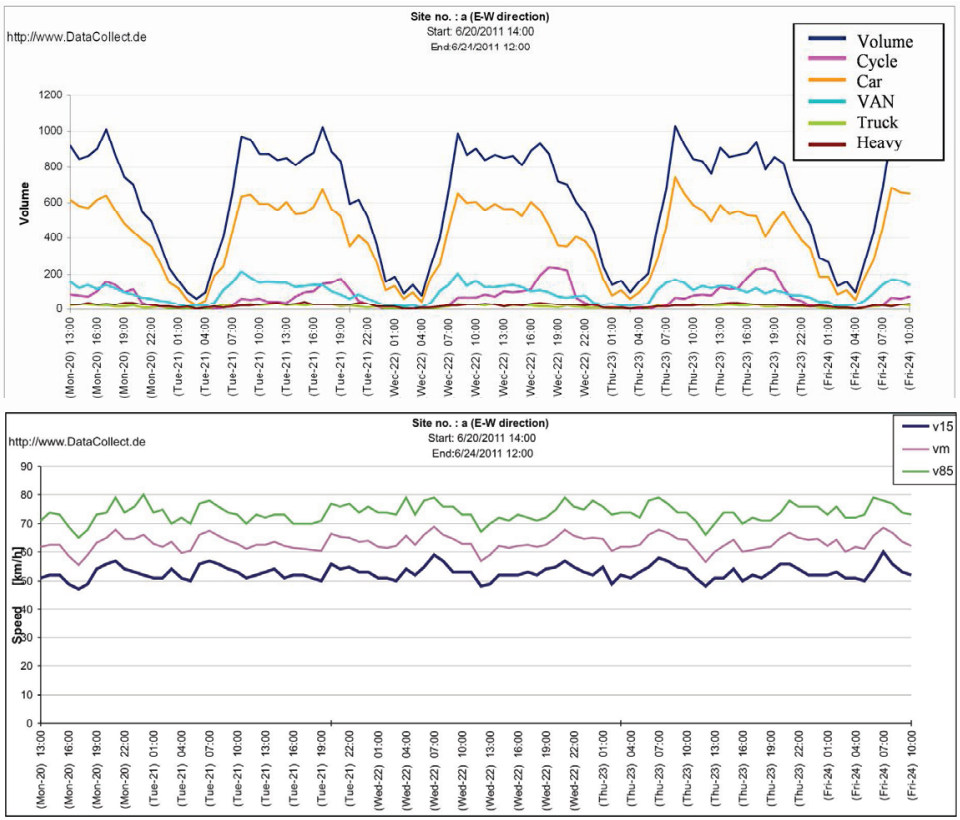

Figure 4: $\quad$ Traffic data results, graphic representation of the vehicles density and characteristics speeds (example for one lane measurements). 


\section{Alternative proposals for access optimisation and the simulation results}

The Syncro 7 software program was used to evaluate the preference of the traffic.

Presently, i.e. without traffic lights, the Junction Capacity Utilization (JCU) for this intersection is of $\mathrm{G}$ level $105.3 \%$ (Figure 5), which constitutes a very high value for such an intersection. It is also important to point out that high JCU values occur at high congestion levels.

A delay of more than 80 seconds/vehicle was found for access to the airport, in both cases, i.e. in and out (Figure 5).

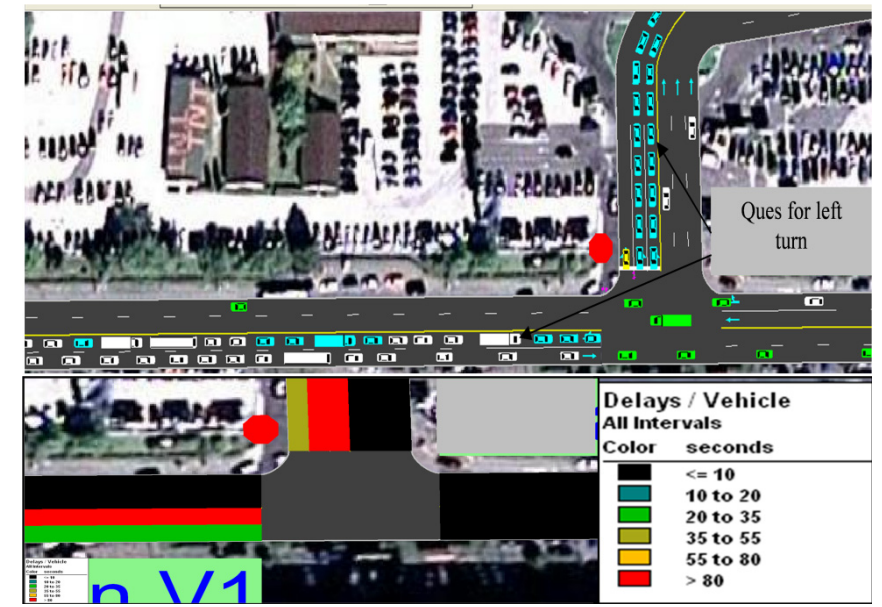

Figure 5: Present traffic condition for the airport entry; Syncro 7 simulation results: the queues and the delay/vehicle estimation.

\subsection{The roundabout design}

The first solution studied was to set up a roundabout with the following characteristics; Circulation line $2 \mathrm{~m}$, Entry radius $20 \mathrm{~m}$, Circulation width $10 \mathrm{~m}$, Island diameter $30 \mathrm{~m}$.

Several development works would need to the carried out in order to build the roundabout, including strengthening of the road and obtaining necessary permissions.

A disadvantage of the roundabout is that it reduces the speed on the main road. Simulation of traffic diverted through the roundabout using Syncro 7 software shows that the intersection used $98.4 \%$ (Figure 6). 


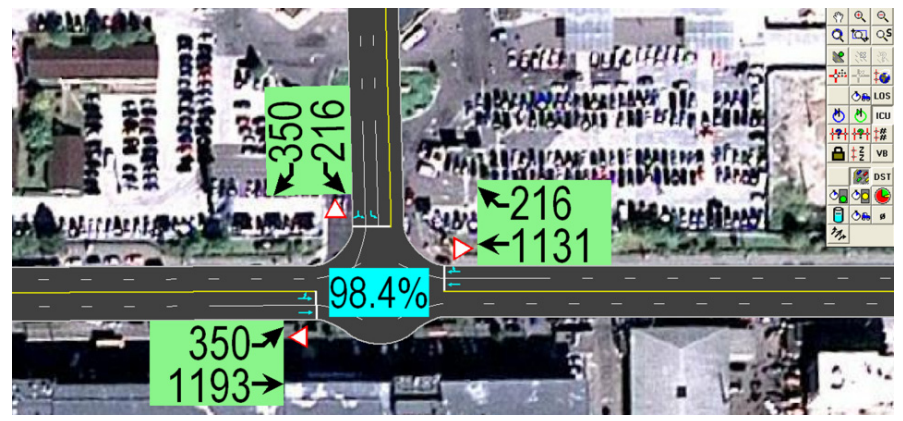

Figure 6: The JCU level for roundabout simulation.

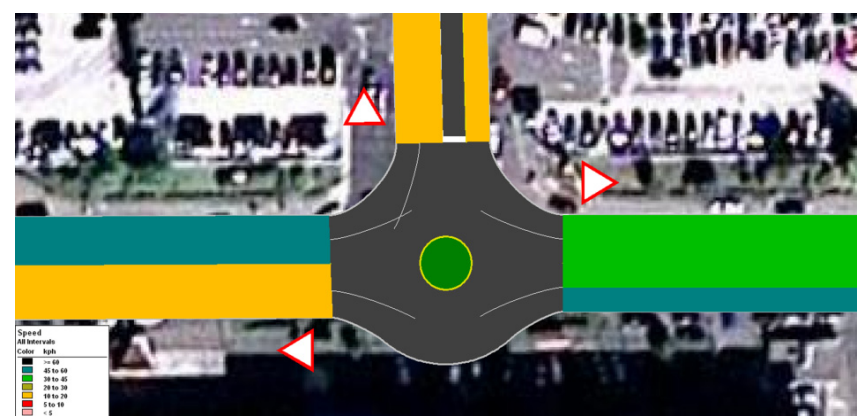

Figure 7: $\quad$ The effect of roundabout on car speed, simulation results.

The main advantage of the roundabout is for vehicles leaving the airport and E60 artery. On the W-E line the traffic becomes congested. This solution greatly reduces the vehicular velocity along the western artery due to the excessive number of vehicles and the waiting time increases (Figure 7).

It has been found that the movement speed in the western lane is of 10 to $20 \mathrm{~km} / \mathrm{h}$, which is too low for a volume of over 1,700 vehicles/hour.

Delays for vehicles in the western lane were 55 to 80 seconds in accordance with the Synhro 7 simulation. These values are lower than in the case of the existing junction but still too high to be accepted as an optimum solution.

\subsection{Design of traffic lights with demand optimisation}

The traffic light system was studied as an alternative to a roundabout. In order to minimise disruptions to the main road traffic, it was proposed to add a turn left lane.

The identification of the number of vehicles turning left (W-N and N-E) was carried out using inductive loops, which are well-tested devices. The first vehicle detection loop was rectangular and positioned $5 \mathrm{~m}$ before the STOP line. The second loop, also rectangular, was located $30 \mathrm{~m}$ from the junction and was designed to detect the length of the waiting queue (Figure 8). 


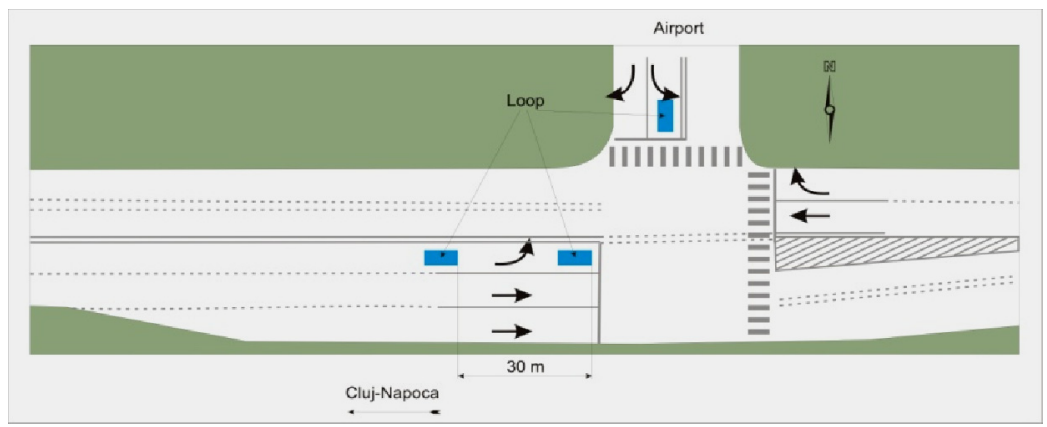

Figure 8: The traffic junction design for traffic lights optimization with request phase.

The traffic light cycle was designed taking into account occasional presence of pedestrians crossing the main road. This gave origin to two designs, depending on the presence or absence of pedestrians (design A).

The second design (or design B) was characterised by including a fixed green light time calculated in accordance to the presence of pedestrians, i.e. 18 seconds.

Another design assumes there are no pedestrians $\left(\right.$ design $\left.\mathrm{B}^{1}\right)$, and has a variable green time of 7 to 20 seconds, depending on the demand, provided that the vehicles are turning left in the direction $\mathrm{N}-\mathrm{W}$. This time - controlled by inductive detectors - has the required value to decrease the length queue at the traffic lights.

There is also the possibility of a demand required by the presence of at least one vehicle in the first loop and lasting 7 to 33 seconds.

Thus the development of the traffic lights will be as follows. Firstly, for Plan A, the traffic light cycle will be fixed with a minimum duration of 45 seconds. In the case of heavy traffic conditions, with constant requests from all directions, the traffic lights will perform the cycle $\mathrm{A}-\mathrm{B}^{1}-\mathrm{C}-\mathrm{A}-\mathrm{B}^{1}-\mathrm{C}$ changing the length of phases $\mathrm{B}$ and $\mathrm{C}$ according to demand. When the request from a certain direction disappears, that phase of the traffic light cycle is skipped until it reoccurs.

The traffic light system was simulated in Syncro 7, which allows for the evaluation of its advantages and disadvantages.

The simulation revealed the following results:

- $\quad$ Service-level intersection is at $\mathrm{C}$ level ( $\mathrm{LOS}=$ Level of Service).

- JCU-level use in this case is $87.4 \%$, level C.

- Traffic light-cycle has a duration of $98 \mathrm{~s}$ (must not exceed $120 \mathrm{~s}$ ).

- The average delay is of 15 seconds.

The pattern of traffic lights time is shown in Figure 9. When the sensors are activated, the simulation gave the following green time values for each lane: EBT-lane (W-E) 45 and 65 seconds of green phase; EBL-lane (W-N) and 20 seconds of green phase; SBL-Lane (N-E) 33 and seconds, which is 


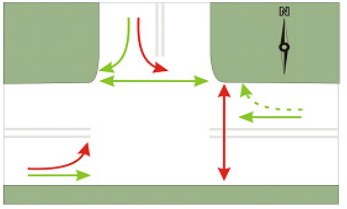

Phase A

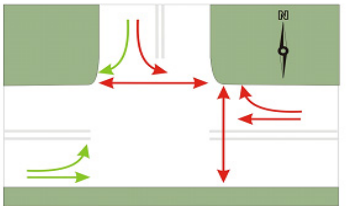

Phase B"

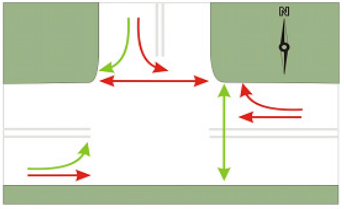

Phase B'

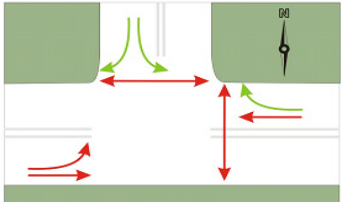

Phase C

Figure 9: The pattern of traffic optimization lights.

superimposed with pedestrian phase; WBT-lane (E-W) 45 seconds; Pedestrian phase is 18 seconds.

Analysing the delay time per vehicle it can be seen that the main arteries (W$\mathrm{E}$ and $\mathrm{E}-\mathrm{W}$ ) are not affected, the delays are less than 10 seconds. This is important considering that through that intersection has been counted a number of over 2500 vehicles per hour.

The traffic light diagram is shown in Figure 10, the line represents a vehicle or a group of vehicles and depending on their movement, the lights will turn green or red.

It can be seen in Figure 10 that a sort of triangle is formed by the parallel and horizontal lines, and it is preferable that the base of this triangle is as narrow as possible because the horizontal line represents vehicle stops. It is noted that the

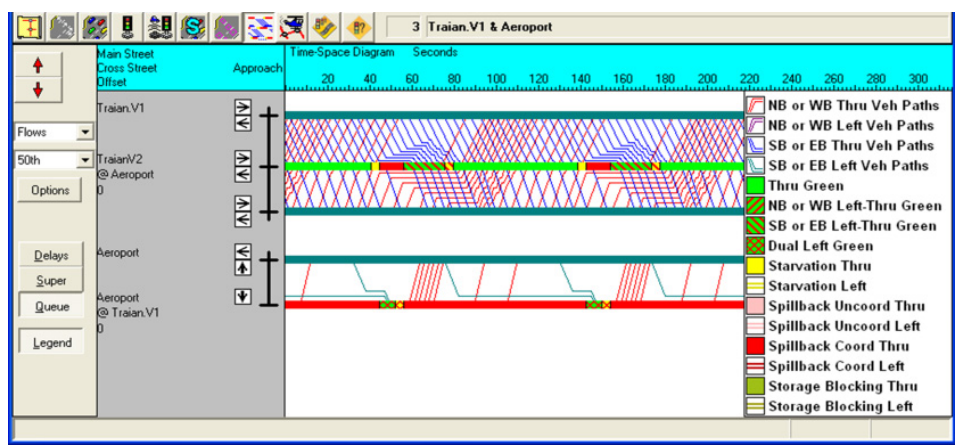

Figure 10: The traffic lights diagram. 
only lanes where the vehicles will be waiting 35 to 55 seconds are the airport in and out lanes (i.e. those equipped with detection sensors). In these cases the waiting time is not long because the number of vehicles is still small and the sensor is set in such a way that the vehicle needs to stop at least five seconds to trigger a request.

\section{Conclusions}

The analysis of the simulation results for the proposed intersection have shown that the optimal solution is the use of traffic lights versus building a roundabout. The justification for the solution is given by the ratio between the number of vehicles accessing the airport (4,834/day) and the total number of vehicles passing through the junction $(27,800 /$ day $)$.

An additional reason to propose the traffic light solution is the added safety that such a system offers. The roundabout design instead would reduce the traffic speed in the roundabout even more and induce an imbalance in one of the directions of travel.

\section{Acknowledgement}

This work was supported by CNCSIS-UEFISCDI, project number PNII-IDEI code $2531 / 2008$.

\section{References}

[1] Akçelik, R. Implementing Roundabout and Other Unsignalized Intersections Analysis Method in SIDRA. Australian Road Research Board, Working Paper WD TE91/002.

[2] Kimber, R.M.; Hollis, E.M., Traffic Queues and Delays at Junctions, Transport and Road Research Laboratory, Report LR 909, Crowthorne, 1979.

[3] Fisk. C. S. Traffic performance analysis at roundabouts. Transportation Research Part B: Methodological, Volume 25, Issues 2-3, April-June 1991, pp. 89-102.

[4] Ding-Wei H. Phase diagram of a traffic roundabout. Physica A: Statistical Mechanics and its Applications Volume 383, Issue 2, 15 September 2007, pp. 603-612.

[5] Tan, J. Estimation of Traffic Queues and Delays at Roundabout Entries. Third International Symposium on Intersections Without Traffic Signals. 1997, pp. 248-260.

[6] Pratelli, A. Souleyrette, R.R. Roundabout perception: review of standards and guidelines for advanced warning, Urban Transport and the Environment in the 21st Century, 2011, pp. 71-82.

[7] Brilon, W. Roundabouts: A State of the Art in Germany. Paper presented at the National Roundabout Conference, Vail, Colorado; 2005. 
[8] National Accident Report 2009-2010. http://www.gestionesinistri.ro /accidente-rutiere-statistica-accidente-rutiere-2009/.

[9] García-Nieto J. Swarm intelligence for traffic light scheduling: Application to real urban areas. Engineering Applications of Artificial Intelligence. http://dx.doi.org/10.1016/ j.engappai.2011.04.011

[10] Hu, X, Wang, W, Sheng. H. Urban Traffic Flow Prediction with Variable Cell Transmission Model. Journal of Transportation Systems Engineering and Information Technology, Volume 10, Issue 4, August 2010, pp. 73-78.

[11] Ebben, M, a.a. Dynamic one-way traffic control in automated transportation systems. Transportation Research Part B: Methodological. Volume 38, Issue 5, June 2004, pp. 441-458.

[12] Traffic controller MT4040 - Programming handbook. Ed. Fontana, Rev. 8, Segrate, 2005 pp. 18-58.

[13] SDR Handbook. DataCollect System GmbH, Germany 2007.

[14] Syncro $7++$ Handbook. Texas, 2006. 